\title{
Investigación de mercado sobre el grado de aceptación de mermelada de cocona en Sibundoy, Putumayo*
}

\section{Market Research on the Degree of Acceptance of Cocona Jam in Sibundoy, Putumayo}

\section{Francisco Emilio Argote \\ Diana Patricia Vargas \\ Héctor Samuel Villada}

Referencia para citar este artículo: ARGOTE, F. E.; VARGAS, D. P. y VILLADA, H. S. (2013). "Investigación de mercado sobre el grado de aceptación de mermelada de cocona en Sibundoy, Putumayo”. En: Revista Guillermo de Ockham 11(2). pp. 197-206.

\section{Resumen}

La cocona es una fruta exótica proveniente de la amazonia colombiana de excelentes características sensoriales y nutricionales. Previene enfermedades cardiovasculares, obesidad, diabetes y ciertos tipos de cáncer. Los objetivos desarrollados en la presente investigación fueron valoración de la demanda potencial de mermelada a partir de cocona, evaluación sensorial, determinación de productos sustitutos y diseño de estrategias de mercadeo. El modelo de investigación empleado fue el cuantitativo probabilístico. La recolección de la información se hizo mediante encuesta. La población objeto de estudio del municipio de Sibundoy correspondió a 2.188 hogares, con una muestra representativa de 327 observaciones. El consumo de la mermelada es trimestral, con cantidades de dos unidades de $200 \mathrm{~g}$. Entre los productos sustitutos se encontraron el bocadillo y el arequipe. La demanda potencial para la mermelada de cocona es de alrededor de 13.063 unidades/año. Los catadores no encontraron diferencias significativas para las variables color, sabor y textura, más sí en la de aroma en la prueba del producto en relación con marcas comerciales.

Palabras clave: fruta, nutracéutico, demanda, catador, sustituto.

- Fecha de recepción del artículo: 22-05-2013 • Fecha de aceptación: 16-07-2013

FRANCISCO EMILIO ARGOTE. Ingeniero agroindustrial. Especialista en docencia universitaria y gerencia de mercadeo. Magíster en Mercadeo Agroindustrial. Doctorando en Recursos y Tecnologías Agroalimentarias. Docente asociado del programa de agroindustria, Universidad de San Buenaventura Cali. Correo electrónico: feargote@usbcali.edu.co. DIANA PATRICIA VARGAS. Ingeniera Agroindustrial. Estudiante de Maestría en Ingeniería Agroindustrial, Universidad Nacional, Palmira. Instructora del Sena regional Putumayo. HÉCTOR SAMUEL VILLADA. Ingeniero Agroindustrial. PhD en Ingeniería. Docente del Programa de Agroindustria, Universidad del Cauca.

Artículo corto derivado del proyecto de aula, realizado por estudiantes de la Maestría en Ingeniería Agroindustrial de la Universidad Nacional de Colombia, sede Palmira. Fecha de inicio: febrero de 2012. Fecha de finalización: agosto de 2012. 


\section{Abstract}

The cocona is an exotic fruit from the colombian Amazon, with excellent sensory and nutritional characteristics, prevents cardiovascular diseases, obesity, diabetes, and certain cancers. The objectives developed in this investigation were assessing the potential demand from cocona jam, sensory evaluation, and determination of substitute products and designing marketing strategies. The research model used was the quantitative probabilistic. The data collection was conducted through a survey. The study population Sibundoy Township homes fell to 2188, with a representative sample of 327 observations. Consumption is jam quarterly amounts of two units of $200 \mathrm{~g}$. Among the substitutes were found the guava and fudge. The potential demand for cocona jam is around 13,063 units / year. Tasters found no significant differences for the variables of color, flavor and texture, but if in the aroma in product testing with trademarks.

Keywords: fruit, nutraceutical, demand, wine, substitute.

\section{Introducción}

La amazonia colombiana es una región que presenta gran biodiversidad en lo referente a frutales; sin embargo, la producción y comercialización es poco conocida a nivel nacional y su cultivo se limita a los departamentos del Caquetá, Putumayo, Guaviare, Guainía y Amazonas (Arco et al., 2004).

Según algunas investigaciones, la cocona (Solanum sessiliflorum dunal) es una fruta exótica con alto contenido de pectina (Muñoz, 2011), antioxidantes y carbohidratos (Cáceres, Andrade y Silva, 2012) y elevada productividad en su cultivo (Silva et al., 2005). Es utilizada normalmente en la agroindustria para la elaboración de productos como agua saborizada, refrescos, bebidas energizantes, complementos vitamínicos, salsas y mermeladas, entre otros (Yuyama et al., 2008) (ver Figura 1).

Por lo anterior, se hace necesaria la implementación de un proceso tecnológico para su agroindustrialización y comercialización, dado que las tendencias de los consumidores se inclinan hacia alimentos con ingredientes activos (Proexport, 2003; Cardona et al., 2011).
Figura 1

Cocona (Solanum sessiliflorum dunal)

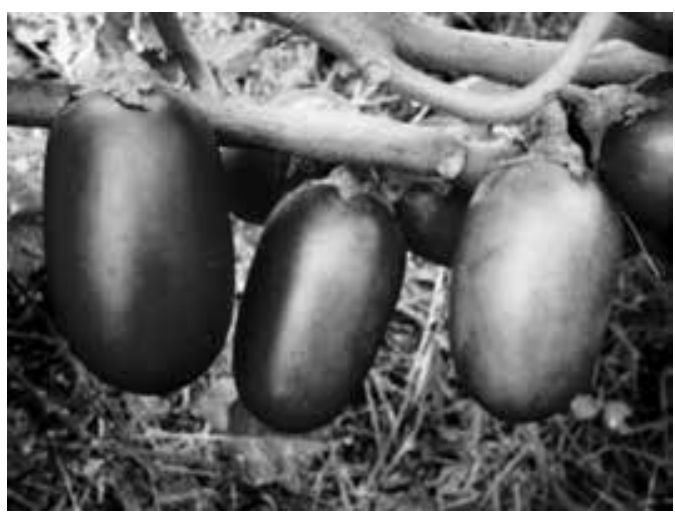

Fuente: http://www.crfg.org

La cocona es una fruta posee un aroma y sabor incomparables y sus características organolépticas hacen de ella un producto agroindustrial excepcional. Por su composición nutricional puede constituirse en una alternativa alimenticia para personas que sufren padecimientos como obesidad, diabetes, enfermedades cardiovasculares y cierto tipos de cáncer (Silva et al.,2010). Adicionalmente, la mermelada a partir de cocona es en un producto nutracéutico y muy competitivo en nichos de mercados internacionales y regionales (Yuyama et al., 2008).

Las mermeladas son productos cuyos ingredientes principales son fruta y azúcar. Pueden conservar algunas características básicas de las materias primas utilizadas en su elaboración, de las cuales el consumidor espera que sean bajas en azúcares y por ende en calorías, situación que ha motivado a la agroindustria para producir mermeladas light a base de frutas nutracéuticas y exóticas (Boatella, Condony y López, 2004).

La investigación de mercados es una herramienta que recolecta y analiza información acerca del comportamiento de los consumidores (Esqueda y López, 2010; Chopra y Meindl, 2007), lo cual permite disminuir la incertidumbre en la toma de decisiones (Mariampolski, 2001; Kotler y Keller, 2006). De esta manera, las empresas investigan los mercados con el fin de efectuar un constante seguimiento a las experiencias, características y motivos de compra del consumidor, así como a las influencias ambientales, culturales, políticas o de otra índole, las cuales influyen sustancialmente en el momento de decidirse por un producto (Jassir, 2009; Tas y Tokmakcioglu, 2010; Pujol, 2002). 
Las pruebas con catadores permiten hacer los ajustes necesarios al prototipo del producto previos a su distribución y comercialización. Para el éxito de estas pruebas, la asociación colombiana de ciencia y tecnología de alimentos (Acta) (2008), recomienda tener en cuenta los siguientes factores, entre otros:

- La muestra debe ser delimitada por personas de la población en general o por un panel entrenado.

- Las pruebas sensoriales pueden ser subjetivas. La degustación se lleva a cabo con la población en general y el cuestionario debe especificar únicamente si le agrada o no el producto y qué le recuerda. Esto se puede considerar como una prueba cualitativa que se puede evaluar con estadística no paramétrica.

- Las pruebas sensoriales objetivas se efectúan con un panel entrenado (mínimo quince personas) el cual debe responder un cuestionario en el que especifique los niveles de las características; por ejemplo sabor dulce, menos dulce, no dulce. Esto resulta en una prueba cuantitativa que se puede evaluar con estadística paramétrica.

- Al escoger los panelistas para una evaluación sensorial es necesario elaborar una encuesta a fin de buscar enfermedades o comportamientos que demuestren que la persona no es apta para identificar sabores por no tener memoria sensorial y por ende no califican para este tipo de pruebas.

- Las instalaciones pueden ser comedores, cocinas, supermercados, paneles, que garanticen la asepsia en la preparación de las muestras y la comodidad de los panelistas.

El objetivo de esta investigación es conocer el grado de aceptación de la mermelada de cocona, por parte de la población del municipio del Sibundoy, departamento del Putumayo, región que hace parte de la amazonia colombiana y en donde se cultiva esta fruta exótica.

\section{Materiales y método}

El modelo de investigación empleado fue el modelo descriptivo con muestreo probabilístico.
La recolección de la información se hizo mediante encuesta diseñada con preguntas filtro, cerradas y de selección múltiple.

\section{Área de estudio}

El Cuadro 1 muestra la población residencial y no residencial beneficiaria del servicio de energía del municipio de Sibundoy y en él se puede apreciar que la población residencial del sector rural asciende a 1.864 hogares, superada por el sector urbano con 4.285 hogares; el centro poblado presenta 291 suscriptores. Por otro lado, los beneficiarios no residenciales ascienden a 43 en el área rural, 163 en la urbana y 16 en el centro poblado (Alcaldía de Sibundoy, 2012; Emevasi, 2012).

Cuadro 1

Población beneficiaria de energía

\begin{tabular}{|l|r|r|r|r|}
\hline \multicolumn{1}{|c|}{ Usuario } & Rural & Urbano & C. Poblado & Total \\
\hline Estrato 1 & 1.863 & 2.137 & 251 & 4.251 \\
\hline Estrato 2 & 1 & 2.113 & 39 & 2.153 \\
\hline Estrato 3 & $\mathrm{ND}$ & 35 & 1 & 36 \\
\hline Estrato 4 & $\mathrm{ND}$ & $\mathrm{ND}$ & $\mathrm{ND}$ & $\mathrm{ND}$ \\
\hline Estrato 5 & $\mathrm{ND}$ & $\mathrm{ND}$ & $\mathrm{ND}$ & $\mathrm{ND}$ \\
\hline Estrato 6 & $\mathrm{ND}$ & $\mathrm{ND}$ & $\mathrm{ND}$ & $\mathrm{ND}$ \\
\hline Residencial & 1.864 & 4.285 & 291 & 6.440 \\
\hline Industrial & 2 & 20 & $\mathrm{ND}$ & 22 \\
\hline Comercial & 1 & 48 & 1 & 50 \\
\hline Oficial & 40 & 91 & 11 & 142 \\
\hline Otros & $\mathrm{ND}$ & 4 & 4 & 8 \\
\hline No residencial & 43 & 163 & 16 & 222 \\
\hline Total & 1.907 & 4.448 & 307 & 6.662 \\
\hline
\end{tabular}

Fuente: Emevasi, 2007

\section{Población objetivo}

La población objetivo de este estudio se encuentra en el área urbana y en el centro del poblado. Se toman como base los hogares de los estratos 2 y 3 cuyo promedio de integrantes se encuentra entre tres y cuatro personas. De acuerdo con lo anterior, la población objetivo alcanzó los 2.188 hogares. 


\section{Cálculo de la muestra}

Se aplicó la fórmula (Pujol, 2012 citado por Argote et al., 2009) para población finita con un nivel de confianza del $95 \%$ y un error del $5 \%$ :

$$
n=\frac{N Z^{2} p q}{(N-1) e^{2}+Z^{2} p q}
$$

donde:

$n=$ tamaño de la muestra.

$N=$ población objetivo.

$Z$ = valor de la tabla normal estándar correspondiente al área bajo la curva.

$p=$ Probabilidad de que un suceso ocurra (generalmente un $50 \%$ ).

$q=$ Probabilidad de que un suceso no ocurra (generalmente un $50 \%$ ).

$e=$ Error permisible

Se efectuaron 327 observaciones distribuidas de manera proporcional en cada uno de los estratos, como lo indica el Cuadro 2

Cuadro 2

Tamaño de la muestra

\begin{tabular}{|l|l|l|l|}
\hline \multirow{2}{*}{ Ubicación } & \multirow{2}{*}{ Hogares } & \multicolumn{2}{c|}{ Encuestas/Estrato } \\
\cline { 3 - 4 } & & \multicolumn{1}{|c|}{ II } & \multicolumn{1}{c|}{ III } \\
\hline Urbano & 2.148 & 316 & 5 \\
\hline C. poblado & 40 & 5 & 1 \\
\hline Total & 2.188 & 321 & 6 \\
\hline
\end{tabular}

\section{Evaluación sensorial}

Adicional a la investigación de mercado, se llevó a cabo una prueba de producto con quince catadores entrenados quienes evaluaron la mermelada de cocona en las variables sabor, color, olor y textura, mediante el empleo de una escala hedónica con puntuación de 1 a 5, donde 1 es pésimo, 2 malo, 3 regular, 4 bueno y 5 excelente. La prueba de producto se desarrolló aplicando la metodología Blind test; es decir, se desconoce la procedencia de las muestras, con "ubicación central" a "tríos" donde se compararon la mermelada de estudio con dos comerciales, así: mermelada de pińa (A), cocona (B) y manzana (C). Se escogieron estos productos dadas sus características sensoriales cercanas a las del estudio. Las mermeladas comerciales fueron de la marca San Jorge. Las muestras se sirvieron sobre porciones de galletas con sal cuyo peso fue de cinco gramos, como se puede apreciar en la Figura 2.

Las calificaciones obtenidas se analizaron con el método estadístico de comparación de muestras pareadas de Kruskal Wallis con un nivel de significancia del 0,05 y la prueba de Tuckey. El programa empleado para el procesamiento de datos fue Statgraphics 5.1.

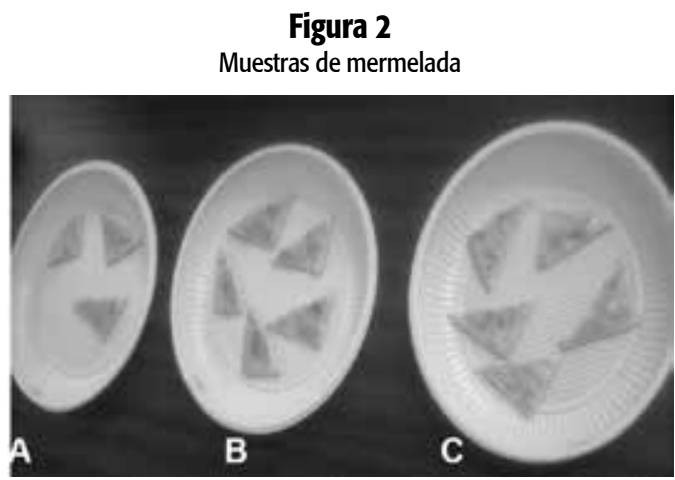

Se diseñaron estrategias enfocadas al marketing mix (producto, precio, plaza promoción y publicidad).

\section{Resultados}

\section{Encuesta}

La frecuencia de consumo de la mermelada revela que es un producto que no hace parte de la canasta familiar (Figura 3) ya que el $87 \%$ de los hogares encuetados consumen mermelada de en cuando, lo cual equivale a una frecuencia trimestral.

\section{Figura 3}

Frecuencia de consumo de mermelada

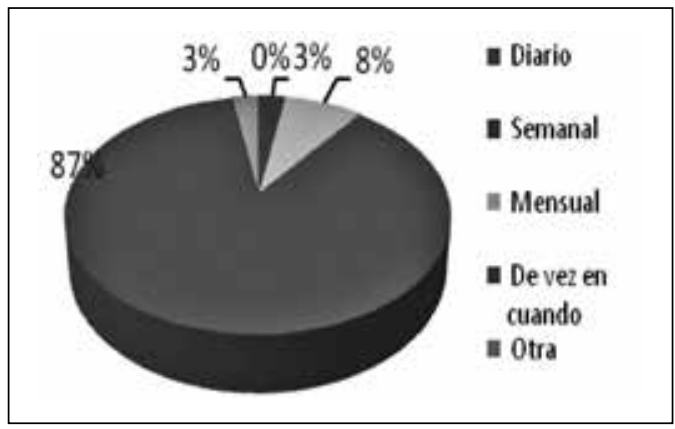


El $53 \%$ de los hogares de los estratos II y III prefieren la presentación de $200 \mathrm{~g}$; el $45 \%$ de 250 g y el $2 \%$ se inclinan por $315 \mathrm{~g}$, como se puede observar en la Figura 4.

Figura 4

Preferencias sobre la presentación

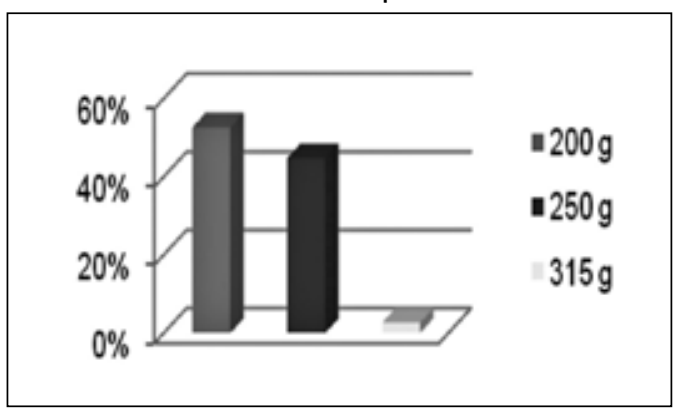

Los hogares encuestados prefieren en un $57 \%$ la presentación de la mermelada en bolsa dosificadora y un $43 \%$ se inclinan por el envase de vidrio (Figura 5). En relación con el precio que están dispuestos a pagar por una mermelada de cualquier sabor este se encuentra entre $\$ 2.001 \mathrm{y}$ $\$ 3.500$, como lo indica el Cuadro 3.

Figura 5

Presentación que prefieren los hogares del municipio de Sibundoy

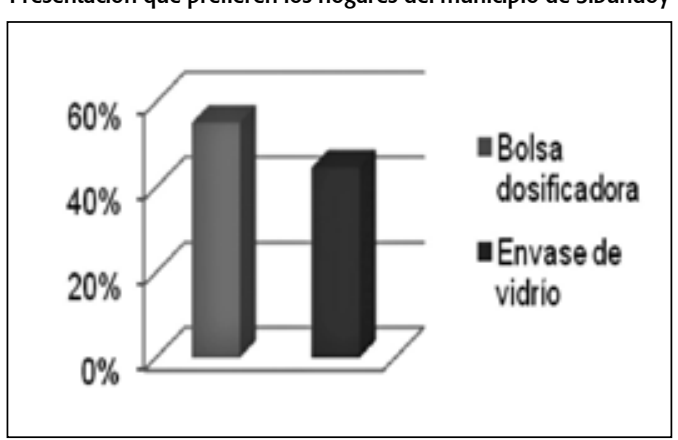

Cuadro 3

Precios de la mermelada comercial de $250 \mathrm{~g}$.

\begin{tabular}{|l|c|}
\hline \multicolumn{1}{|c|}{ Oscilación de precios } & Población \\
\hline$\$ 1000$ a $\$ 2000$ & $26 \%$ \\
\hline$\$ 2001$ a $\$ 3500$ & $71 \%$ \\
\hline$\$ 3501$ a $\$ 6000$ & $3 \%$ \\
\hline
\end{tabular}

Los hogares identificaron varias razones de compra entre las que se encuentran:

- Sabor agradable.

- Salud.

- Presentación.
El producto fue escogido por su sabor agradable en un $73 \%$ de los casos, el cual es un porcentaje representativo. Para el $24 \%$ de los hogares encuestados la presentación del producto también es importante. Otras características importantes sugeridas para el producto son la inclusión de trocitos de frutas y la reutilización del envase. Para tres de cada cien hogares del municipio de Sibundoy la salud no es una razón para escoger una marca en particular.

Los principales lugares de compra de las mermeladas son tiendas y supermercados, de los cuales estos últimos son los lugares de mayor preferencia, como se puede apreciar en el Cuadro 4. Los supermercados como Super Sibundoy y tiendas como Doña Ofelia son los lugares más frecuentados para comprar mermeladas debido a que tienen los precios más bajos del mercado.

\section{Cuadro 4}

Lugares de compra de las mermeladas

\begin{tabular}{|l|c|}
\hline \multicolumn{1}{|c|}{ Lugares } & Preferencia \\
\hline Tienda & $24 \%$ \\
\hline Supermercado & $76 \%$ \\
\hline
\end{tabular}

Es importante tener en cuenta que el municipio de Sibundoy es un lugar retirado y en algunas épocas la mermelada escasea, razón por la cual la población se ve obligada a consumir otros productos como compotas, bocadillos, jaleas, confites de fruta, arequipe y frutas en almíbar (Cuadro 5). Estos productos constituyen los sustitutos de la mermelada cuando por diferentes factores como la escasez y el precio, entre otros, aquella no se puede adquirir. El arequipe y el bocadillo son los principales sustitutos de la mermelada en presentaciones de $125 \mathrm{~g}$ y $500 \mathrm{~g}$.

Por otra parte, se puede apreciar mediante el presente estudio que los hogares presentan una clara intención de compra por la mermelada de cocona al observar que el $75 \%$ de los encuestados se inclina por productos innovadores fabricados a partir de frutas exóticas de la amazonia, con precios que fluctúan entre $\$ 2.001$ y $\$ 3.500$ para presentaciones de $250 \mathrm{~g}$. Por otra parte, el interés se inclina hacia las propiedades nutracéuticas y en lo referente al empaque las opiniones están divididas, ya que más de la mitad de los hogares prefiere la bolsa dosificadora y el resto el envase de vidrio. 


\section{Cuadro 5}

Productos sustitutos de la mermelada

\begin{tabular}{|l|c|c|}
\hline \multicolumn{1}{|c|}{ Producto } & Presentación $(\mathrm{g})$ & Consumo $\%$ \\
\hline Compotas & $110-113$ & 5 \\
\hline Bocadillo & $125-250$ & 24 \\
\hline Jaleas & $250-500$ & 3 \\
\hline Confites de fruta & $4-5$ & 8 \\
\hline Arequipe & $125-500$ & 53 \\
\hline Frutas en almíbar & $125-500$ & 7 \\
\hline
\end{tabular}

\section{Porcentaje de participación de mermeladas en el mercado del municipio de Sibundoy}

Con base en la población objetivo del estudio la cual correspondió a 2.188 hogares y tomando el trimestre como frecuencia de consumo de la mermelada, se llevó a cabo el análisis de participación respecto a los sustitutos, como se puede apreciar en el Cuadro 6.

\section{Cuadro 6}

Participación de la mermelada respecto a sustitutos

\begin{tabular}{|l|l|l|}
\hline \multicolumn{1}{|c|}{ Producto } & \multicolumn{1}{|c|}{$\begin{array}{c}\text { Cantidad } \\
\text { estimada } \\
\text { Unidades/ańo }\end{array}$} & Participación \% \\
\hline Compotas & 10.502 & 7 \\
\hline Bocadillo & 75.617 & 48 \\
\hline Jaleas & 1.575 & 0.1 \\
\hline Confites de fruta & 25.206 & 16 \\
\hline Arequipe & 27.831 & 18 \\
\hline Frutas en almíbar & 1.838 & 1.2 \\
\hline Mermelada & 15.229 & 10 \\
\hline
\end{tabular}

La fórmula empleada para realizar los cálculos de la demanda fue la siguiente:

Demanda real $=\%$ de hogares consumidores $\mathrm{x}$ población $\mathrm{x}$ cantidad de consumo $\mathrm{x}$ frecuencia.

Demanda real de mermeladas $=87 \% \times 2.188$ $\mathrm{x} 2$ unidades $\mathrm{x} 4$ trimestres $=15.229$ unidades/año.

Es importante señalar que el consumo de mermelada anual de los hogares encuestados es bajo comparado con los principales sustitutos antes mencionados. Por tal razón es necesario hacer sondeos de mercado en otros departamentos de Colombia y en el exterior para determinar las potencialidades del producto fuera del área de estudio de esta investigación.

\section{Cálculo de la demanda potencial para las presentaciones de mermelada de cocona de 200 g, 250 g y 315 g}

La demanda potencial de las diferentes presentaciones se calculó teniendo en cuenta la intención de compra de los hogares (1.641 hogares que corresponden al $75 \%$ ) y la preferencia de presentación de acuerdo con la Figura 4. Los resultados fueron los siguientes:

Demanda potencial $=\%$ de aceptación de consumidores $\mathrm{x}$ población $\mathrm{x}$ cantidad de consumo $\mathrm{x}$ frecuencia.

- Presentación de $200 \mathrm{~g}=53 \%$ x 1.641 x 2 × 4 $=6.958$ unidades/año.

- Presentación de 250 g (45 \%) = 5.908 unidades/año.

- Presentación de 315 g (3\%) = 197 unidades/ año.

Para garantizar la demanda potencial de la mermelada de cocona, se deben tener en cuenta las presentaciones de $200 \mathrm{~g}$ y $250 \mathrm{~g}$, las cuales son asequibles para los hogares de los estratos 2 y 3 . Es importante anotar que la población objeto de estudio es pequeña y se toma como referencia para determinar los grados de percepción y aceptación del producto; sin embargo, se observa una clara tendencia de los hogares hacia el consumo de productos nutracéuticos.

\section{Evaluación sensorial}

En el Cuadro 7 se muestran los resultados obtenidos mediante el método estadístico de Kruskall Wallis, el cual evalúa la hipótesis nula consistente en que las medianas dentro de cada uno de los datos de los tres tipos de mermeladas son iguales o al menos un par de los tipos de mermeladas presenta diferencias significativas teniendo en cuenta el valor de P.

Para las variables sabor, color y textura, el valor de $\mathrm{P}$ es mayor que 0.05 . No existe, entonces, una diferencia estadísticamente significativa entre las medianas con un nivel del $95.0 \%$ de confianza para las tres muestras de mermelada.

En la variable aroma, el valor de $P$ es menor que 0.05 , lo cual indica una diferencia estadísticamente significativa entre las medianas con un nivel del $95.0 \%$ de confianza. 
Cuadro 7

Prueba de Kruskal-Wallis para mermelada de piña, cocona y manzana

\begin{tabular}{|l|c|c|c|c|}
\hline \multirow{2}{*}{ Mermelada } & \multicolumn{4}{|c|}{ Rango promedio } \\
\cline { 2 - 5 } & Sabor & Color & Textura & Aroma \\
\hline Pińa & 26.5 & 22.7 & 25.4 & 29.6 \\
\hline Cocona & 23.3 & 26.9 & 21.8 & 17.6 \\
\hline Manzana & 19.4 & 19.4 & 21.8 & 21.8 \\
\hline Estadístico & 2.57231 & 3.58857 & 1.08308 & 10.7905 \\
\hline Valor P & 0.276332 & 0.16625 & 0.581852 & 0.0045381 \\
\hline
\end{tabular}

En las figuras de cajas y bigotes, la parte rectangular de la gráfica se extiende desde el cuartil inferior hasta el cuartil superior y cubre la mitad central de cada muestra. La línea central dentro de cada caja indica la localización de la mediana de cada muestra. Los bigotes se extienden desde la caja hasta los valores máximo y mínimo en la muestra.

También se incluyen en la gráfica muescas que cubren una distancia arriba y abajo de la mediana. Cuando las muescas de cualquier par de medianas se traslapan, no existe una diferencia estadísticamente significativa entre ellas, con un nivel de confianza del $95 \%$. Si las muescas de cualquier par de medianas no se traslapan, existe una diferencia estadísticamente significativa entre ellas.

Los resultados de los diagramas de cajas y bigotes se pueden observar en las Figuras 6, 7, 8 y 9 para las variables de sabor, color, textura y aroma, respectivamente.

\section{Estrategias de mercado}

Las siguientes estrategias formuladas buscan comunicar y resaltar las características nutricionales del producto y posicionar la nueva mermelada de cocona en el municipio de Sibundoy.

Figura 6

Diagrama de cajas y bigotes en sabor

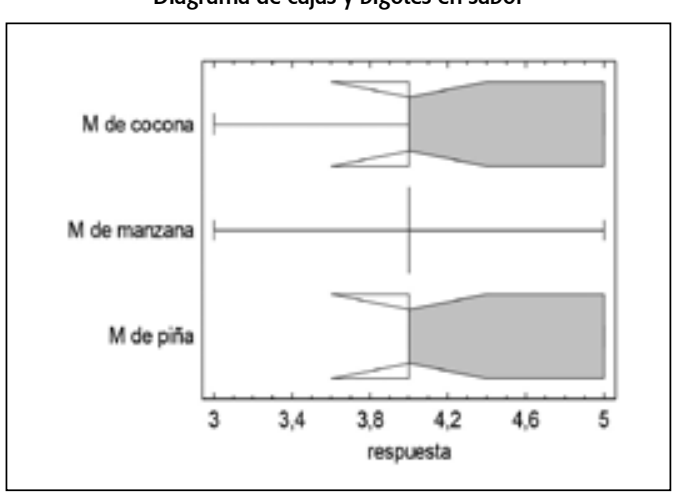

Figura 7

Diagrama de cajas y bigotes en color

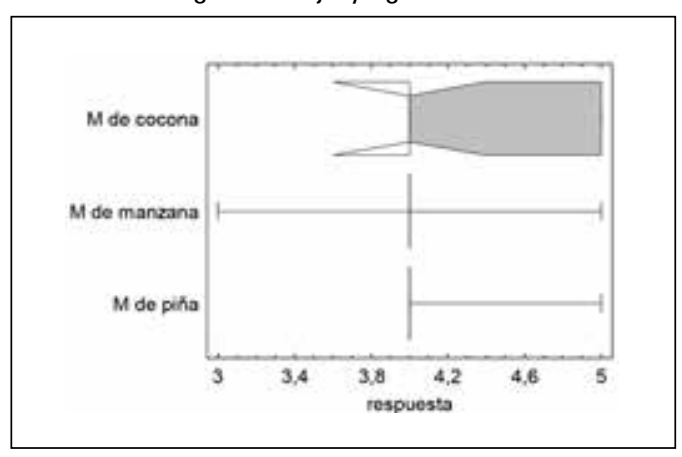

Figura 8

Diagrama de cajas y bigotes en textura

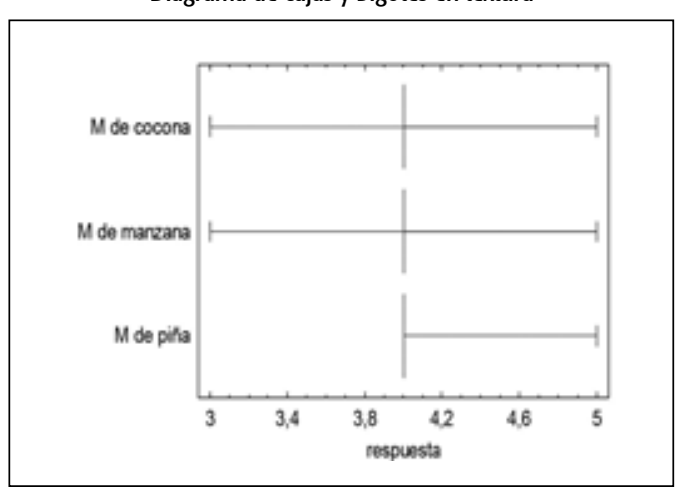

Figura 9

Diagrama de cajas y bigotes en aroma

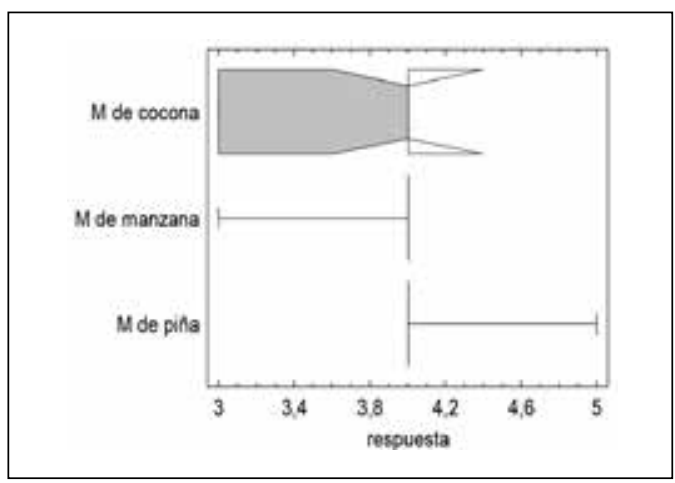

\section{Estrategias respecto al producto}

Dos tipos de presentaciones, a saber, bolsa dosificadora y envase de vidrio con el fin de acoger satisfactoriamente a las sugerencias de los clientes.

La información técnica dará relevancia al nombre del producto: ingredientes, fecha de vencimiento, instrucciones de conservación, uso, contenido, lote, fecha de vencimiento y registro sanitario, con lo cual se cumple con la normativa 
nacional que establece el Invima y se garantiza que es un producto elaborado en condiciones asépticas.

Mediante folletos informativos de recetas que acompañarán al producto, se indicarán las cantidades necesarias de mermelada de cocona para acompañar carnes y preparar postres.

\section{Estrategias respecto al precio}

- Inicialmente el precio del producto puede ser de penetración, teniendo en cuenta las recomendaciones de los clientes potenciales.

- La mermelada se puede comercializar en compañía de otros productos exóticos, como galletas de quinua y chachafruto, entre otros, especialmente para mercados internacionales con descuentos por ventas al por mayor.

\section{Estrategias respecto de la plaza}

- Se llevará la mermelada a las góndolas de tiendas y supermercados donde exista la mayor concentración de clientes y se ubicará en la línea de productos gourmet.

- Efectuar sondeos de mercado en las principales ciudades del país y en Estados Unidos

\section{Estrategias respecto a la comunicación}

- Publicitar a través de la televisión y la radio las frutas amazónicas, como la cocona, para resaltar sus bondades nutricionales y medicinales.

- Es importante emprender el mercadeo electrónico de este tipo de productos exóticos. Los diseños de las páginas deben permitir a los clientes potenciales interactuar virtualmente con el producto mediante juegos, documentales, fondos de escritorio para el computador y concursos, entre otros.

- Dadas las características sensoriales y medicinales del producto los niños pueden ser un mercado objetivo para este. Por ello se recomienda una educación a temprana edad que enseñe las bondades de las frutas exóticas provenientes de la amazonia.
- Aunado a lo anterior, se debe diseńar un plan de comunicación acorde con los perfiles del cliente revelados en la inteligencia de mercados a fin de incursionar en el exterior con marcas de origen.

\section{Conclusiones}

La encuesta a la población reveló que esta adquiere la mermelada de $200 \mathrm{~g}$ con poca frecuencia, lo que permite concluir que este producto no hace parte de la canasta familiar. No obstante, las empresas productoras de mermeladas de frutas exóticas pueden dar a conocer las características nutracéuticas de este tipo de alimentos, así como como elaborar mermeladas dietéticas que puedan hacer parte de de la línea de alimentos saludables.

Se recomienda incursionar otros mercados para la comercialización de la mermelada en los departamentos vecinos, como Nariño, Huila y Cauca, mediante la implementación de las estrategias mencionadas de plaza, publicidad y promoción.

El precio de adquisición de la mermelada sin especificar sabor, fluctúa entre los $\$ 2.001$ y los $\$ 3.500$. Para mermeladas elaboradas a partir de frutas desconocidas a nivel nacional, se deben diseñar estrategias de comunicación para que el producto sea conocido y logre el posicionamiento adecuado en la mente del consumidor.

En cuanto al empaque, la mitad de la población de los hogares encuestados del municipio de Sibundoy prefiere el envase de vidrio reutilizable, ya que la cultura del ama de casa aún sigue vigente respecto a esta presentación. La otra mitad defiende la bolsa dosificadora por ser este un empaque que disminuye los riesgos de contaminación física en caso de ruptura, aligera el peso en el transporte y ocupa menos espacio en la nevera o en la alacena.

El producto es ofertado en supermercados y tiendas, lugares en los cuales debe hacerse una mayor promoción y publicidad ya que la población prefiere las mermeladas de frutas convencionales como mora, pińa y fresa de marcas como San Jorge y Fruco y solo una pequeña proporción de consumidores (alrededor del $3 \%$ ) adquiere mermeladas de empresas putumayenses como Agroinpa. El gusto es una característica dominante 
en el momento de adquirir las mermeladas en la población objeto de estudio, en tanto que en las grandes ciudades y en otros países, las mermeladas de frutas exóticas como cocona, arazá, copoazú y camu camu son ofertadas en casas naturistas por sus propiedades nutraceúticas.

La mermelada tiene dos productos sustitutos, a saber, el bocadillo y el arequipe (este último con características de consumo similares a las de la mermelada) que podrían ser mezclados para elaborar productos innovadores.

En cuanto a los resultados del Blind test evaluado con la prueba de Kruskal-Wallis, estos demostraron que no existe diferencia estadística significativa entre las medianas de los datos para las variables sabor, color y textura. Lo anterior se explica dado que la aceptación de la mermelada de cocona respecto a las características de sabor, color y textura arrojó como resultado rangos promedios de 23.3, 26.9 y 21.8 respectivamente. El color por su parte, tiene un rango promedio más alto respecto a las otras muestras, razón por la cual puede convertirse en una característica llamativa que sirva de pauta para diseñar un empaque que permita apreciarla de manera notable.

El aroma fue la característica con un valor $\mathrm{P}$ menor que 0.05 ; o sea, con diferencia estadísticamente significa y rango promedio bajo (17.6) en la prueba de Kruskal-Wallis, información corroborada por los diagramas de cajas y bigotes. Este es un resultado esperado dado que el aroma de la cocona es fuerte y no es propio de las frutas de clima frío ni de las comerciales como mora, granadilla y banano, entre otras. Para facilitar su introducción en el mercado se recomienda diseñar estrategias efectivas de mercado a fin de que la fruta sea conocida por la población de Sibundoy.

\section{Bibliografía}

- ALCALDÍA DE SIBUNDOY (2012). Ajuste del esquema de ordenamiento territorial (EOT). Sibundoy, Colombia.

- ARCO, A., BECERRA, M., BENITEZ, A. y DÍAZ, J. (2004). Desarrollo de biocomercio sostenible en la región amazónica colombiana (proyecto). Bogotá, Colombia: Instituto de investigación de recursos biológicos Alexander Von Humboldt.

- ARGOTE, F., VILLADA, H. y ARGOTE, H. (2009). Investigación de mercado sobre el grado de aceptación de la carne de cuy (Cavia Porcellus) en presentaciones de ahumado, croquetas y apanado en la ciudad de Pasto. En: Revista Biotecnología en el sector agropecuario y agroindustrial 7(1). pp. 55-66.

- ASOCIACIÓN COLOMBIANA DE CIENCIA Y TECNOLOGÍA DE ALIMENTOS (ACTA) (2008). Estadistica aplicada en evaluación sensorial. Bogotá, Colombia.

- BOATELLA, J., CODONY, R. y LOPEZ, P. (2004). Química y bioquímica de los alimentos II. vol. 1. Barcelona, España: Universitat de Barcelona.

- CÁCERES, L., ANDRADE, J. y SILVA, D. (2012). Effects of peeling methods on the quality of cubiu fruits. En: Revista Ciencia y Tecnología de Alimentos 2(3). pp. 255- 260.

- CARDONA, J., CUCA, L. y BARRERA, J. (2011). Determinación de algunos metabolitos secundarios en tres tipos de cocona (Solanum sessiliflorum Dunal). En: Revista Colombiana de Química 40(2). pp. 185-200.

- CHOPRA, S. y MEINDL, P. (2007). Supply Chain Management, Strategy, Planning, and Operations. New York, United States: Prince Hall.

- EMPRESA DE ENERGÍA DEL VALLE DE SIBUNDOY EMEVASI. (2012). Registro de hogares suscritos a Emevasi. Sibundoy, Colombia.

- ESQUEDA, S. y LÓPEZ, S. (2010). Investigación de mercado en Venezuela: la opinión de los expertos. En: Revista Debates IESA 11(2). pp. 32-45 
- JASSIR, E. (2009). Neuroimágenes en la investigación de mercados. En: Revista Pensamiento y gestión 26(1). pp. 73-93.

- KOLTLER, P. y KELLER, K.(2006). Marketing management. New Jersey, United States: Prentice Hall.

- MARIAMPOLSKI, H. (2001). Qualitative Market Research: A Comprehensive Guide. California, United States: Thousand Oaks.

- MUÑOZ, F. (2011). Extracción y caracterización de la pectina obtenida a partir del fruto de dos ecotipos de cocona (Solanum sessiliflorum), en diferentes grados de madurez, a nivel de planta piloto. [M.Sc Ingeniería Agrícola] Colombia: Universidad Nacional de Colombia. Facultad de Ciencias Agrí́colas.

- PROEXPORT COLOMBIA E INSTITUTO ALEXANDER VON HUMBOLDT. (2003). Estudio de mercado, frutas amazónicas en el estado de California, Estados Unidos. Bogotá, Colombia.

- PUJOL, B. (2002). Dirección de marketing y ventas. Madrid, Espańa: Cultura S.A.

- SILVA FILHO, D., YUYAMA, L., AGUIAR, J., OLIVEIRA, M. y MARTINS, L. (2005). Caracterização e avaliaçáo do potencial agronômico e nutricional de etnovariedades de cubiu (Solanum sessiliflorum Dunal) da Amazônia. En: Revista Acta amazonica 35(4). pp. 399-405.

- SILVA, M., AGUIAR, J., SOUZA, F., CARVALHO, Y. y YUYAMA, L. (2010). Processamento e avaliaçáo da farinha de cubiu em diferentes condiçoes de armazenamento. XIX Jornada de Iniciação Científica PIBIC INPA.

- TAS, O. y TOKMAKCIOGLU, K. ( 2010). Efficient market hypothesis and comovement among emerging markets. En: Revista Doğus Üniversitesi Dergisi 2(11). pp. 286-301.

- YUYAMA, L., PANTOJA, L., MAEDA, R., AGUIAR, J. y SILVA, S. (2008). Desenvolvimento e aceitabilidade de geléia dietética de cubiu (Solanum sessiliflorum Dunal). En: Revista Ciencia y Tecnología de Alimentos 28(4). pp. 929-934. 